\title{
P112 Influence of Cuff Blood Pressure Accuracy on Identification of Isolated Systolic Hypertension
}

Dean Picone ${ }^{1, *}$, Martin Schultz ${ }^{1}$, Matthew Armstrong ${ }^{1}$, Willem Bos ${ }^{2,3}$, Nathan Dwyer ${ }^{4,5}$, Peter Lacy ${ }^{6}$, Esben Laugesen ${ }^{7}$, Stefano Omboni ${ }^{8,9}$, Giacomo Pucci ${ }^{10}$, Philip Roberts-Thomson ${ }^{5,1}$, George Stouffer ${ }^{11}$, Kenji Takazawa ${ }^{12}$, Thomas Weber ${ }^{13}$, Berend Westerhof ${ }^{14}$, James Sharman ${ }^{15}$

${ }^{1}$ Menzies Institute for Medical Research, College of Health and Medicine, University of Tasmania, Australia

${ }^{2}$ St Antonius Hospital, Department of Internal Medicine, Nieuwegein, The Netherlands

${ }^{3}$ Department of Internal Medicine, Leiden University Medical Center, Leiden, The Netherlands

${ }^{4}$ Menzies Institute for Medical Research, College of Health and Medicine, University of Tasmania, Hobart, Australia

${ }^{5}$ Royal Hobart Hospital, Hobart, Australia

${ }^{6}$ UCL Institute of Cardiovascular Science

${ }^{7}$ Department of Endocrinology and Internal Medicine, Aarhus University Hospital, Aarhus, Denmark

${ }^{8}$ Clinical Research Unit, Italian Institute of Telemedicine, Varese, Italy

${ }^{9}$ Scientific Research Department of Cardiology, Science and Technology Park for Biomedicine, Sechenov First Moscow State Medical University,

Moscow, Russian Federation

${ }^{10}$ Unit of Internal Medicine at Terni University Hospital, Department of Medicine, University of Perugia, Perugia, Italy

${ }^{11}$ Division of Cardiology, University of North Carolina at Chapel Hill, Chapel Hill, United States

${ }^{12}$ Center for Health Surveillance and Preventive Medicine, Tokyo Medical University Hospital, Tokyo, Japan

${ }^{13}$ Cardiology Department, Klinikum Wels-Grieskirchen, Wels, Austria

${ }^{14}$ Department of Pulmonary Diseases, VU University Medical Center, Amsterdam, The Netherlands

${ }^{15}$ Menzies Institute for Medical Research, College of Health and Medicine, University of Tasmania, Hobart, Australia

\section{ABSTRACT}

Introduction: Isolated systolic hypertension (ISH) is the most common form of hypertension in older people. However, accurate identification of ISH may be hindered because cuff blood pressure (BP) underestimates systolic BP (SBP) and overestimates diastolic BP (DBP). This study aimed to determine the influence of cuff BP accuracy on the identification of ISH.

Methods: Cuff BP and invasive aortic BP were measured simultaneous (or near-simultaneously) in 1737 subjects ( $63 \pm 12$ years, $68 \%$ male) during coronary angiography. Data was derived from 32 studies, using 20 different cuff BP devices, from the Invasive Blood Pressure Consortium (INSPECT). ISH was defined as $\geq 140 /<90 \mathrm{mmHg}$ according to cuff BP and invasive aortic BP.

Results: According to cuff BP, $25 \%$ of subjects $(n=430)$ had ISH, however, $37 \%(n=648)$ were identified with ISH from invasive aortic BP. There was $77 \%$ concordance between cuff and invasive BP for identifying ISH. Among the $23 \%(n=408)$ of subjects misclassified by cuff BP, $38 \%(n=155)$ of misclassification was from SBP underestimation (mean: $-16.6,95 \%$ CI: -18.9 to $-13.9 \mathrm{mmHg})$, whereas $35 \%(n=143)$ was from DBP overestimation $(15.6,11.9$ to $19.0 \mathrm{mmHg})$ and $20 \%(n=83)$ from SBP overestimation (17.6, 14.4 to $20.5 \mathrm{mmHg}$ ). Subjects misclassified were on average 2.7 years older and had greater body mass index $\left(0.8 \mathrm{~kg} / \mathrm{m}^{2}\right)$ than those correctly classified.

Conclusion: Approximately one quarter of older subjects have ISH misclassified, mostly because of underestimation of cuff SBP and overestimation of cuff DBP. This demonstrates a need to improve the accuracy of cuff BP methods for greater precision in identifying ISH.

(C) 2019 Association for Research into Arterial Structure and Physiology. Publishing services by Atlantis Press International B.V. This is an open access article distributed under the CC BY-NC 4.0 license (http://creativecommons.org/licenses/by-nc/4.0/). 